\title{
Magnetic-Field-Based 3D ETREE Modelling for Multi-Frequency Eddy Current Inspection
}

\author{
Yu Zhang ${ }^{1}$ and Yong $\mathrm{Li}^{2}$ \\ ${ }^{1}$ Engineering College, Air Force Engineering University, \\ ShaanXi, Xi' an, 710038, P.R. China \\ octzy@qq. com \\ ${ }^{2}$ School of Aerospace, Xi' an Jiaotong University, \\ ShaanXi, Xi' an, 710049, P.R. China \\ yong.li@mail.xjtu.edu.cn
}

\begin{abstract}
More and more solid-state magnetic field sensors such as Hall devices are used in eddy current inspection (EC) to acquire magnetic field signals. This work extends the previous analytical model, i.e. 2D Extended Truncated Region Eigenfunction Expansion (ETREE) of EC, and focuses on establishment of 3D ETREE of multi-frequency eddy current inspection (MFEC) on stratified conductors, while taking into account the solid-state magnetic field sensors for field quantification and rectangular coils for field excitation. 3D Finite Element Modelling (FEM) and a hybrid modelling are adopted for verification of the established model. It has been noticed that the 3D ETREE implements the fast and accurate computation of magnetic field signals of MFEC. Following that, the directional characteristics of EC with rectangular excitation coils are investigated, which reveals that the coil width contributes more to the measurement sensitivity than the coil length, and benefits the evaluation of defects and anisotropic conductivity profile of conductors in the follow-up study.
\end{abstract}

Keywords: Eddy current inspection, Magnetic field, Extended Truncated Region Eigenfunction Expansion, Finite element modelling.

\section{Introduction}

To evaluate the integrity and structural health of metallic structures such as stratified conductors, Electromagnetic Non-destructive Evaluation (ENDE) techniques, especially Eddy Current (EC) and transient eddy current, are preferred and used in realtime inspections [1].

Eddy-current testing traditionally relies on the detection of impedance changes in a pickup coil as it moves across the inspected specimen. Accordingly, the theoretical modelling for EC previously was implemented merely to predict: (1) the impedance signals from the stranded induction coils for time-harmonic field [2], [3]; and (2) the electromotive force (EMF) signals from coils for transient field [4]. However, it is formidable for traditional EC to detect deep flaws in conductive materials, because low frequency excitation is demanded to allow eddy currents to penetrate deeply into the conductors while the sensitivity of normal pickup coils, which is proportional to 
the excitation frequency, is decreased. In light of this, it is more advantageous to measure the magnetic field using solid-state magnetic field sensors such as Hall sensors, giant magnetoresistive (GMR) sensors, or superconducting quantum interference devices (SQUIDs) [5], [6] are used.

The theoretical modelling for EC in conjunction with solid-state magnetic field sensors has been conducted for years. Ward and Moulder proposed an expression for transient EC signals to layered conductive structures using Hall device based on the infinite integral formulation developed by Dodd and Deeds [7]. However, the sensing element in Hall devices was not taken into account. Li and Tian extended the Truncated Region Eigenfunction Expansion (TREE) modelling and made it capable of predicting the magnetic field signals while the dimension of the sensing element was considered [8], [9], [10]. Nevertheless, the model was only applicable to circular coils rather than rectangular coils.

This paper elaborates the formulation of expressions of 3D magnetic field for Directional Eddy Current Inspection (DEC) with rectangular coils via 3D Extended Truncated Region Eigenfunction Expansion modelling (3D ETREE), which concerns the geometry of rectangular coils as well as the dimension of sensing elements. The rest of the paper is organised as follows: Section 2 presents the formulation of the closed-form expressions of magnetic field signals from solid-state magnetic field sensors; the verification of 3D ETREE by comparing with simulation results from 3D Finite Element Modelling (FEM) and a hybrid modelling is exhibited in Section 3; Section 4 elaborates the analysis of directional characteristics of DEC via investigation of the influence of the length and width of a rectangular coil on the measurement sensitivity of DEC to conductivity variation in a conductive halfspace.

\section{Theoretical Background}

The 3D ETREE modelling using Second-order Vector Potential (SOVP) formulation is conducted with a rectangular coil $\left(x_{1} \neq y_{1}, x_{2} \neq y_{2}\right)$ placed over the layered conductive sample which is shown in Figure 1. The rectangular coil, with $N$ number of windings, is supposed to be supplied with a pulsed excitation current, each harmonic of which is written as $I\left(\omega_{i}\right)$ at the angular frequency of $\omega_{i}$.

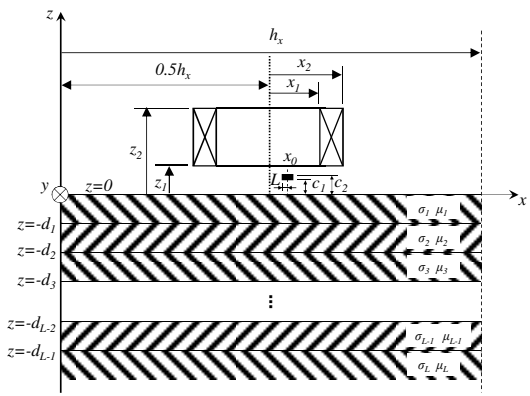

(a)

Fig. 1. A rectangular coil and a magnetic field sensor placed over a multilayered conductor: (a) side view in $\mathrm{y}$-direction; (b) side view in $\mathrm{x}$-direction 


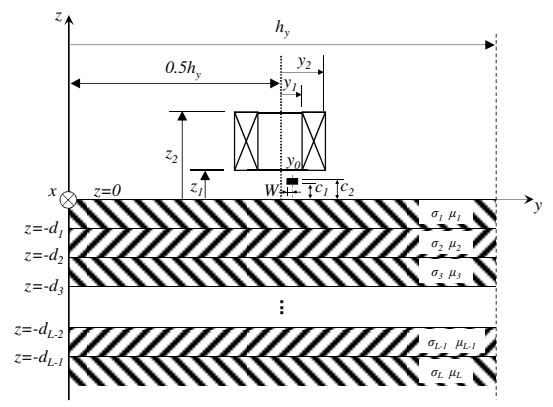

(b)

Fig. 1. (continued)

\subsection{Magnetic Field at a Point of $(x, y, z)$}

Using SOVP formulation, the magnetic vector potential can be written as [1]:

$$
\bar{A}=\nabla \times\left(W_{a} \bar{z}_{0}+\bar{z}_{0} \times \nabla W_{b}\right) .
$$

Thus, magnetic field between the bottom of the rectangular coil and the upper surface of the conductor $\left(0 \leq z \leq z_{1}\right)$ can be expressed as [2]:

$$
\bar{B}=\nabla\left(\frac{\partial W_{a}}{\partial z}\right)
$$

Similar to 2D ETREE modelling, the infinite problem region in 3D is truncated and recast with finite lengths of $h_{x}$ and $h_{y}$ in $x$ and $y$ directions, respectively, which can be seen in Figure 1. The truncation results in the replacement of the infinite integrals with series Eigenfunction expansions. The Eigenvalues $\left(k_{x n}\right.$ and $\left.k_{y m}\right)$ in two truncation directions are computed by applying the boundary condition $\bar{B}=0$ at $z=0, x=h_{x}$ and $y=h_{y}$, which gives [2]:

$$
k_{x n}=\frac{n \pi}{h_{x}}, k_{y m}=\frac{m \pi}{h_{y}}, h_{x} \neq h_{y} .
$$

The magnetic field for each harmonic in the pulsed excitation current is written as:

$$
\bar{B}\left(\omega_{i}\right)=\sum_{m=1}^{\infty} \sum_{n=1}^{\infty}\left[\begin{array}{l}
\sin \left(k_{x n} x\right) \sin \left(k_{y m} y\right) \\
\times\left(e^{\lambda_{m n} z}+e^{-\lambda_{m n} z} \frac{V_{m n, 1}}{U_{m n, 1}}\right) \\
\times C_{m n}\left(k_{x n} \bar{x}_{0}+k_{y m} \bar{y}_{0}+\bar{z}_{0}\right)
\end{array}\right] .
$$


where, $\bar{x}_{0}, \bar{y}_{0}$ and $\bar{z}_{0}$ are unit vectors. $C_{m n}$ denotes the coil coefficient for rectangular coils, which is written as:

$$
\left\{\begin{array}{c}
C_{m n}=\frac{8 \mu_{0} \chi_{m n}\left(e^{-\lambda_{m n} z_{1}}-e^{-\lambda_{m n} z_{2}}\right) N I\left(\omega_{i}\right)}{\left(z_{2}-z_{1}\right) c h_{x} h_{y} k_{x n} k_{y m} \lambda_{m n}} \sin \left(\frac{k_{x n} h_{x}}{2}\right) \sin \left(\frac{k_{y m} h_{y}}{2}\right) \\
\chi=\frac{\sin \left[\left(k_{x n}-k_{y m}\right) c+k_{x n} x_{1}-k_{y m} y_{1}\right]-\sin \left(k_{x n} x_{1}-k_{y m} y_{1}\right)}{2\left(k_{x n}-k_{y m}\right)} \\
-\frac{\sin \left[\left(k_{x n}+k_{y m}\right) c+k_{x n} x_{1}+k_{y m} y_{1}\right]-\sin \left(k_{x n} x_{1}+k_{y m} y_{1}\right)}{2\left(k_{x n}+k_{y m}\right)} \\
c=x_{2}-x_{1}=y_{2}-y_{1}
\end{array} .\right.
$$

$V_{m n, I} / U_{m n, I}$ is the conductor reflection coefficient in the region between the bottom of the coil and the upper surface of the conductor, which is computed by using the iterative equation:

$$
\left\{\begin{array}{l}
U_{m n, j}=\left(\frac{\gamma_{m n, j-1}}{\mu_{j-1}}-\frac{\gamma_{m n, j}}{\mu_{j}}\right) e^{-2 \gamma_{m n, j}\left(d_{j}-d_{j-1}\right)} V_{j+1}+\left(\frac{\gamma_{m n, j-1}}{\mu_{j-1}}+\frac{\gamma_{m n, j}}{\mu_{j}}\right) U_{j+1} \\
V_{m n, j}=\left(\frac{\gamma_{m n, j-1}}{\mu_{j-1}}+\frac{\gamma_{m n, j}}{\mu_{j}}\right) e^{-2 \gamma_{m n, j}\left(d_{j}-d_{j-1}\right)} V_{j+1}+\left(\frac{\gamma_{m n, j-1}}{\mu_{j-1}}-\frac{\gamma_{m n, j}}{\mu_{j}}\right) U_{j+1} \\
\gamma_{m n, j}=\sqrt{\lambda_{m n}^{2}+j \omega_{i} \mu_{j} \sigma_{j}} ; \lambda_{m n}=\sqrt{k_{x n}^{2}+k_{y m}^{2}} \\
U_{m n, L}=\frac{\gamma_{m n, L-1}}{\mu_{L-1}}+\frac{\gamma_{m n, L}}{\mu_{L}} ; V_{m n, L}=\frac{\gamma_{m n, L-1}}{\mu_{L-1}}-\frac{\gamma_{m n, L}}{\mu_{L}} \\
\left.\gamma_{m n, j}\right|_{j=0}=\lambda_{m n} \\
\left.d_{j}\right|_{j=0}=0
\end{array}\right.
$$

The subscript $j$ iterates from $L-1$ to 1 .

\subsection{Integral of Magnetic Field over a Sensor Volume}

Suppose the volume of the sensor element is $2 L \times 2 W \times\left(c_{2}-c_{1}\right)$. The location of the sensor is at the point of $\left(x_{0}, y_{0}, c_{0}\right), c_{0}=\left(c_{2}+c_{1}\right) / 2$. Magnetic field within the sensor element, which is placed between the bottom of the rectangular coil and the upper surface of the first layer of the conductor can be expressed in 3D as:

$$
\bar{B}_{v}\left(\omega_{i}\right)=\frac{\int_{v} \bar{B}\left(\omega_{i}\right) d v}{4 L W\left(c_{2}-c_{1}\right)}=\frac{\int_{x} \int_{y} \int_{z} \bar{B}\left(\omega_{i}\right) d x d y d z}{4 L W\left(c_{2}-c_{1}\right)} .
$$


Two types of sensor dimensions are considered in the model: cubiod shape and cylindrical shape. For a cubiod-shaped sensor with dimension of $2 L \times 2 W \times\left(c_{2}-c_{l}\right)$, Eq. (7) is rewritten as:

$$
\bar{B}_{v}\left(\omega_{i}\right)=\frac{\sum_{m=1}^{\infty} \sum_{n=1}^{\infty} \frac{C_{m n} F_{m n} G_{m n}}{k_{x n} k_{y m}}\left(k_{x n} \bar{x}_{0}+k_{y m} \bar{y}_{0}+\bar{z}_{0}\right)}{L W\left(c_{2}-c_{1}\right)} .
$$

where,

$$
\left\{\begin{array}{l}
F_{m n}=\sin \left(k_{x n} L\right) \sin \left(k_{x n} x_{0}\right) \sin \left(k_{y m} W\right) \sin \left(k_{y m} y_{0}\right) \\
G_{m n}=\left[1+\frac{V_{m n, 1}}{U_{m n, 1}} e^{-\lambda_{m n}\left(c_{2}-c_{1}\right)}\right] \cdot \frac{e^{\lambda_{m n} c_{2}}-e^{\lambda_{m n} c_{1}}}{\lambda_{m n}}
\end{array} .\right.
$$

For cylindrical-shaped sensor with dimension $\pi R^{2} \times\left(c_{2}-c_{1}\right), R=L=W$, Eq. (7) is modified as:

$$
\bar{B}_{v}\left(\omega_{i}\right)=\frac{\sum_{m=1}^{\infty} \sum_{n=1}^{\infty} \frac{C_{m n} E_{m n} G_{m n}}{k_{x n} k_{y m}}\left(k_{x n} \bar{x}_{0}+k_{y m} \bar{y}_{0}+\bar{z}_{0}\right)}{R\left(c_{2}-c_{1}\right)} .
$$

where,

$$
E_{m n}=\left\{\begin{array}{l}
{\left[\cos \left(k_{x n} x_{0}\right) H_{1}\left(k_{x n} R\right)+\sin \left(k_{x n} x_{0}\right) J_{1}\left(k_{x n} R\right)\right]} \\
\times\left[\cos \left(k_{y m} y_{0}\right) H_{1}\left(k_{y m} R\right)+\sin \left(k_{y m} y_{0}\right) J_{1}\left(k_{y m} R\right)\right]
\end{array}\right\} .
$$

It is noted that $E_{m n}, F_{m n}$ and $G_{m n}$ are the sensor coefficients, which depend on the sensor dimensions.

Eqs. (8)-(11) give the expressions of averaged magnetic field within a sensor volume, which can be used for predicting the magnetic field signals acquired from solidstate magnetic field sensors. Following the formulation of field in frequency domain, the discrete time-domain signal i.e. the transient EC response to stratified conductors can be recovered and expressed in the Fourier manner as [9]:

$$
\bar{B}_{v}\left(t_{i}\right)=\frac{1}{M} \sum_{k=0}^{M-1} \bar{B}_{v}\left(\omega_{i}\right) e^{\frac{2 \pi j}{M} k i} k=0,1,2, \ldots, M-1 .
$$

where, $e^{\frac{2 \pi j}{M}}$ is a primitive M'th root of unity. It is noteworthy that Eq. (12) can be efficiently calculated by using MATLAB routine 'ifft' based on Inverse Fast Fourier Transform. 


\section{Corroboration}

In an effort to verify the 3D ETREE modelling, Finite Element Modelling (FEM) is used to simulate the field signals from a cubiod-shaped sensor, when a rectangular coil is placed over a conductive plate. The sensor is placed at the centre of the rectangular coil and $1 \mathrm{~mm}$ above the upper surface of the plate. The dimension of the sensor element is: $L=W=0.9 \mathrm{~mm} ; c_{2}-c_{1}=0.5 \mathrm{~mm}$. The parameters of the coil are listed in Table 1 . The conductivity and relative permeability of the conductive plate are $37 \mathrm{MSm}^{-1}$ and 1 , respectively.

Table 1. Parameters of the excitation coil

\begin{tabular}{cccc}
\hline Coil length & Coil width & Coil height & Winding thickness \\
$2 x_{2} / \mathrm{mm}$ & $2 y_{2} / \mathrm{mm}$ & $z_{2}-z_{1} / \mathrm{mm}$ & $y_{2}-y_{1} / \mathrm{mm}$ \\
\hline 19 & 16 & 7 & 5 \\
\hline Design Lift-off & Number of turns & Excitation & Current in the coil \\
$c_{1} / \mathrm{mm}$ & $N$ & frequency & $I / \mathrm{mA}$ \\
\hline 2 & 500 & $10 \mathrm{~Hz}-10 \mathrm{kHz}$ & 500 \\
\hline
\end{tabular}

The verification is conducted via simulations in frequency domain. The number of elements (NOE) used in FEM is 48608 and the number of degrees of freedom (NOD) is 207437. The $z$-component of magnetic field is acquired and compared. The real and imaginary parts of the field signals against frequency are compared between ETREE and FEM. The results are shown in Figure 2(a). The ETREE results with and without consideration of the dimension of sensor element are shown in Figure 2(b).

The evaluation of agreement in the two comparison cases is realised by using Normalised Root Mean Square Deviation (NRMSD). The computed NRMSD values are listed in Table 2.
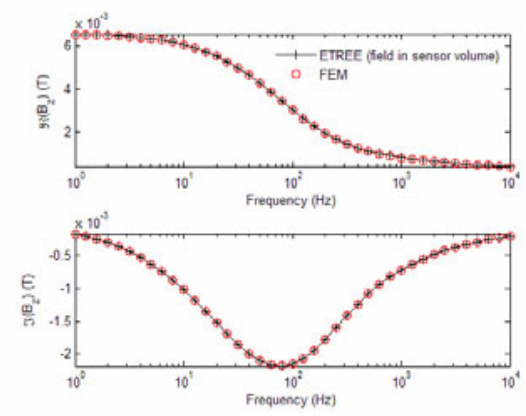

(a)
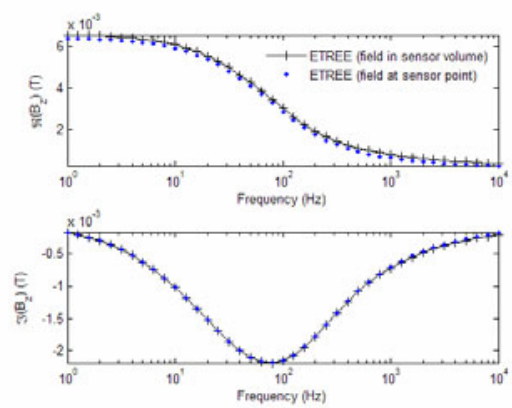

(b)

Fig. 2. Comparison of the magnetic field signal (z-component) predicted by ETREE and FEM: (a) Case 1: ETREE considering sensor dimension vs. FEM; (b) Case 2: ETREE considering sensor dimension vs. ETREE predicting field at sensor point 
Table 2. Computed NRMSD values for the two comparison cases

\begin{tabular}{|c|c|c|c|c|}
\hline \multirow[b]{2}{*}{ NRMSD } & \multicolumn{2}{|c|}{$\begin{array}{l}\text { Case 1: ETREE considering sensor } \\
\text { dimension vs. FEM }\end{array}$} & \multicolumn{2}{|c|}{$\begin{array}{l}\text { Case 2: ETREE considering sensor } \\
\text { dimension vs. ETREE predicting } \\
\text { field at sensor point }\end{array}$} \\
\hline & $\begin{array}{c}\text { Real part } \\
0.20 \%\end{array}$ & $\begin{array}{c}\text { Imaginary part } \\
0.17 \%\end{array}$ & $\begin{array}{c}\text { Real part } \\
2.48 \%\end{array}$ & $\begin{array}{c}\text { Imaginary part } \\
0.57 \%\end{array}$ \\
\hline
\end{tabular}

It can be seen in Figure 2(a) and Table 2 that the predicted signals from sensors by using 3D ETREE have good agreement with that acquired from FEM simulations, since the NRMSD values are much less than $1 \%$, which indicates less residual variance between results from 3D ETREE and FEM. It is also note worthy that the computation time of 3D ETREE (less than 2s) is much less than that of FEM (4 hours) due to large number of NOE and high NOD. Therefore, 3D ETREE can be found advantageous over FEM in terms of high computation accuracy and less simulation time, which facilitates the forward modelling and inverse modelling of EC with rectangular coils.

Figure 2(b) and Table 2 also presents the comparison results regarding 3D ETREE with and without consider the sensor dimension in the modelling. The discrepancy is up to $2.5 \%$, which indicates that the sensor dimension should be taken into account if higher modelling accuracy is pursued, especially in the case where the dimension of sensor is comparable to that of the excitation coil.

\section{Sensitivity Study for DEC}

Following the verification of 3D ETREE, the sensitivity is investigated for DEC with pulsed excitation. The maximum amplitude of the pulsed current is $500 \mathrm{~mA}$. The cycle and the rising time constant of the current are $5 \mathrm{~ms}$ and $100 \mu \mathrm{s}$, respectively. The peak value (PV) against different conductivities of an isotropic conductive half-space is simulated with reference to various dimension of the rectangular coil. Thanks to the high-efficiency 3D ETREE, the database depicting the relation of PV with different dimensions of rectangular coils can be readily established.

In order to obtain PV, the conductivity of the half-space varies and is written as: $\sigma_{i}=\sigma_{0}\left(1+\Delta \sigma_{i}\right)$, where, $\sigma_{0}=37 \mathrm{MSm}^{-1} ; \Delta \sigma_{i}$ varies from $-1 \%$ to $1 \%$. The PVs are extracted in transient EC differential signals $\Delta B_{z i}$, after subtracting the individual signals $B_{z i}$ (when $\Delta \sigma_{i} \neq 0$ ) into the reference signal $B_{z(\sigma 0)}\left(\right.$ when $\Delta \sigma_{i}=0$ ). For a particular rectangular coil with the length $y=19.5 \mathrm{~mm}$ and the width $x=6 \mathrm{~mm}$, the PV against $\Delta \sigma_{i}$ is presented in Figure 3(a). Figure 3(b) shows the plot with errorbars indicating the variation in the curve when (1) the coil length is increased to $21.45 \mathrm{~mm}$; (2) the coil width is increase to $6.6 \mathrm{~mm}$.

It can be seen in Figure 3(a) that the curve of PV exhibits good linearity when $\Delta \sigma_{i}$ varies in the small region from $-1 \%$ to $1 \%$. The slope of the curve $\left(k_{a b}=\mathrm{d} P V / \mathrm{d} \Delta \sigma_{i}\right)$, which is -0.1 in Figure 3(a) indicates the measurement sensitivity to the variation in the sample conductivity. High value of curve slope implies that high measurement sensitivity, which gives significant variation in PV due to the change in conductivity. The curve slope is found varying with the coil dimension, which can be seen in Figure 3(b). 


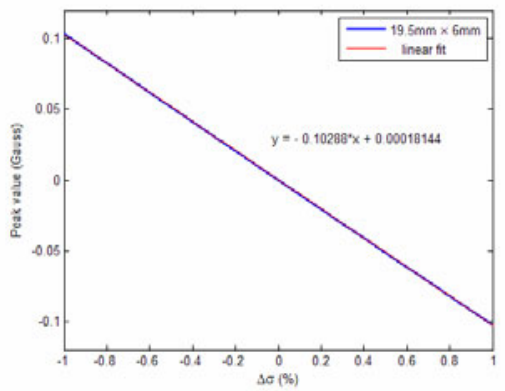

(a)

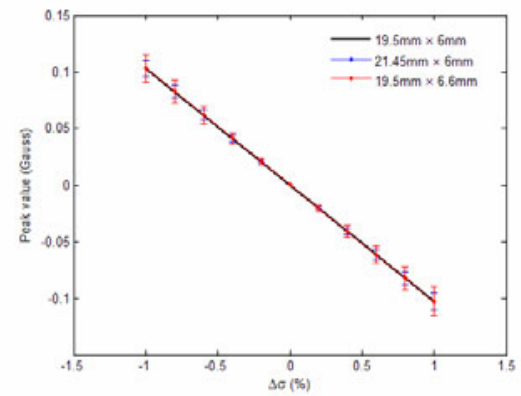

(b)

Fig. 3. PV against $\Delta \sigma_{i}$ for (a) the coil $(x=19.5 \mathrm{~mm}$ and $y=6 \mathrm{~mm})$ and (b) coils $(x=19.5 \mathrm{~mm}$ and $y=6 \mathrm{~mm} ; x=21.45 \mathrm{~mm}$ and $y=6 \mathrm{~mm} ; x=19.5 \mathrm{~mm}$ and $y=6.6 \mathrm{~mm})$

The measurement sensitivities against different combinations of $x$ and $y$ of the coil $(6 \mathrm{~mm} \leq x \leq y \leq 19.5 \mathrm{~mm})$ are modelled. In order to investigate the influences of $x$ and $y$ on the measurement sensitivity, surface fitting using a quadratic function is implemented after the database ( $k_{a b}$ vs. coil dimension) is built up. The results are presented in Figure 4.

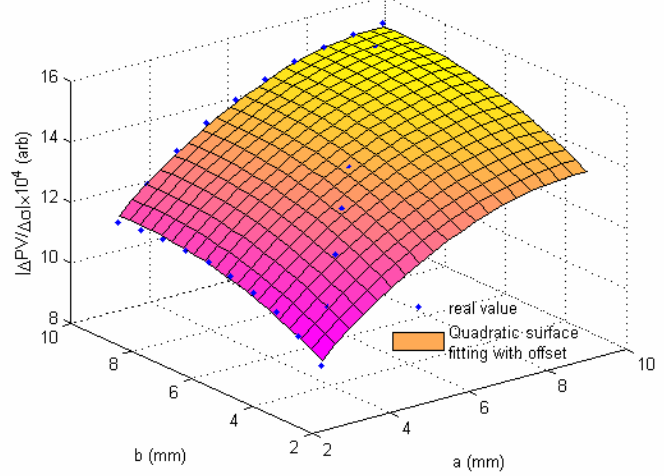

Fig. 4. The measurement sensitivity vs. coil dimension

The quadratic fitting function is written as:

$$
k_{a b}=y\left(8.455 \times 10^{-2}-4.659 y\right)+x\left(1.682 \times 10^{-1}-8.662 x\right)+3.248 \times 10^{-4} .
$$

The fitted curve has $98 \%$ agreement with the database, which is acceptable for the investigation. The influence of $x$ and $y$ on the sensitivity $k_{a b}$ can subsequently be analysed via taking first-order derivative of $k_{a b}$ with respect to $x$ as well as $y$, which can be written as: $\kappa_{x}=\partial\left(k_{a b}\right) / \partial x$ and $\kappa_{y}=\partial\left(k_{a b}\right) / \partial y$. Since the magnitudes of $\kappa_{x}$ and $\kappa_{y}$ are of interest, the absolute values of $\kappa_{x}$ and $\kappa_{y}$ are computed and shown in Figure 5. 


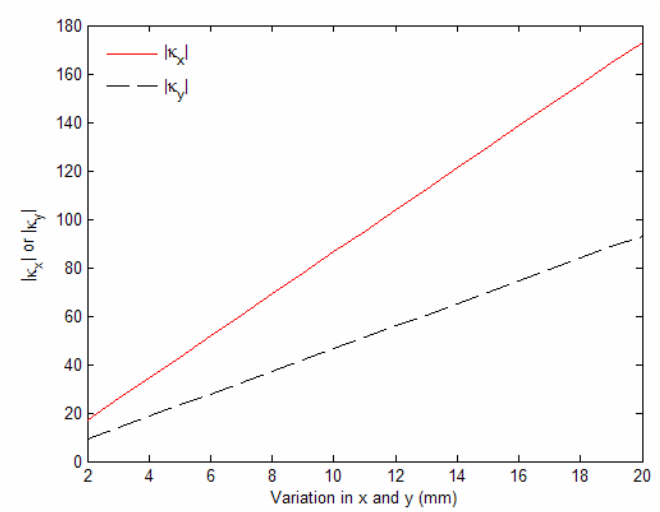

Fig. 5. Influence of $x$ and $y$ on the sensitivity $k_{a b}$ : plots of $\kappa_{x}=\partial\left(k_{a b}\right) / \partial x$ and $\kappa_{y}=\partial\left(k_{a b}\right) / \partial y$

It can be found in Figure 2 that the width $(x)$ plays more important role than the length $(y)$ regarding the contribution to the measurement sensitivity to conductivity variation. The reason lies in the fact that the distance between eddy currents flowing oppositely under the two lengths of the coil varies with the coil width. Small width results in more cancellation of eddy currents. Since eddy currents induce the secondary magnetic field, which is depends on the conductivity of the sample, the cancellation of eddy currents causes decrease of measurement sensitivity. As can be seen in Figure 5, when the width increases, the rate of enhancement of measurement sensitivity rises, which is because less eddy currents are cancelled out. Although the increase in coil length enhances the measurement sensitivity due to increased distance between eddy currents flowing oppositely under the two coil widths, the enhancement due to the coil length is less than that due to the coil width. This is because of the fact that the length is larger than the width, and thus the eddy current density under the coil width is higher than that under the coil length. Therefore, the dependency of measurement sensitivity on the coil length is less than that on the coil width.

Compared with circular coils whose sensitivity is dependent on the coil diameter, the rectangular coils show the distinct characteristics of measurement sensitivity, which is mostly influenced by the coil width. The contribution to the sensitivity from the coil width is more than that from the coil length. As a result, EC with a rectangular coil shows the directional measurement sensitivity, which is along the width of the coil. In such case, DEC is realised, which is advantageous over traditional EC in terms of (1) high detectability of cracks with different orientations; (2) better characterisation of anisotropic distribution of conductivity due to applied stress or strain in particular directions.

\section{Conclusion}

This paper presents the 3D ETREE modelling for prediction of magnetic field signals from solid-state magnetic field sensors, which can be placed at an arbitrary location between bottom of the excitation and the upper surface of the conductor. The consideration of sensor dimension in the formulation results in the new coefficient in the 
expression. The so-called sensor coefficient is dependent on the sensor geometry, and indispensable when (1) EC with utilisation of magnetic field sensor arrays is simulated; (2) the sensor dimension is comparable to coil size.

3D ETREE facilitates the investigation of directional characteristics of measurement sensitivity i.e. change in PV due to conductivity variation in samples for DEC. The influences of the coil width and length on the DEC sensitivity is analysed by setting up a database depicting the relation between measurement sensitivity and coil dimension. It can be noticed that the contributions from the length $(x)$ and the width (y) to the measurement sensitivity are not identical. The coil width is dominant in enhancement of measurement sensitivity. Therefore, compared with traditional EC with circular coils, which has the measurement sensitivity equally on the circumference of the probes, DEC is found having the dominant measurement sensitivity along the coil width, which benefits the evaluation and characterisation of natural cracks and anisotropic conductivity due to stress/strain.

The experimental work on verification of 3D ETREE and the directional characteristics of DEC, which has been found in theoretical study, is currently underway. Further work would be focused on the identification, characterisation and reconstruction of natural defects and inhomogeneous conductivity profiles of conductors.

Acknowledgement. The authors would like to thank Miss. Xinhua Li and Prof. Gui Yun Tian of Newcastle University, UK for 3D FEM simulation and valuable discussions.

\section{References}

1. Sundararaghavan, V., Balasubramaniam, K., Babu, N.R., Rajesh, N.: A multi-frequency eddy current inversion method for characterizing conductivity gradients on water jet peened components. NDT\&E Int. 38, 541-547 (2005)

2. Theodoulidis, T.P., Kriezis, E.E.: Eddy Current Canonical Problems (with Applications to Nondestructive Evaluation). TechScience Press (2006)

3. Theodoulidis, T.P., Bowler, J.R.: Eddy current interaction with a right-angled conductive wedge. Proc. of the Royal Society of London A 461, 3123-3139 (2005)

4. Yang, H.C., Tai, C.C.: Pulsed eddy-current measurement of a conducting coating on a magnetic metal plate. Mea. Sci. \& Tech. 13, 1259-1265 (2002)

5. Tian, G.Y., Sophian, A.: Study of magnetic sensors for pulsed eddy current techniques. Insight. 47, 277-280 (2005)

6. Tian, G.Y., Sophian, A., Taylor, D., Rudlin, J.: Multiple sensors on pulsed eddy-current detection for 3D subsurface crack assessment. IEEE Sensors Journal 5, 90-96 (2005)

7. Ward, W.W., Moulder, J.C.: Low frequency, pulsed eddy currents for deep penetration. Rev. of Prog. in QNDE 17, 291-298 (1998)

8. Li, Y., Theodoulidis, T.P., Tian, G.Y.: Magnetic field-based eddy current modelling for multilayered specimen characterization. IEEE Trans. on Mag. 43, 4010-4015 (2007)

9. Li, Y., Tian, G.Y., Simm, A.: Fast analytical modelling for pulsed eddy current evaluation. NDT \& E Int. 41(6), 477-483 (2008)

10. Tian, G.Y., Li, Y., Mandache, C.: Study of lift-off invariance for pulsed eddy-current signals. IEEE Trans. on Mag. 45, 184-191 (2009) 